\title{
Genome-wide identification and expression analysis of glycine-rich protein genes in Chinese cabbage (Brassica rapa L. ssp. pekinensis)
}

Xiaonan Lu

Shanxi Agricultural University

Ming Gao

Shanxi Agricultural University

Yaxiong Cheng

Shanxi Agricultural University

Meilan Li

Shanxi Agricultural University

Xiaoyong XU ( $\nabla$ xuxy7926@163.com )

Shanxi Agricultural University https://orcid.org/0000-0001-8691-9437

Research article

Keywords: Chinese cabbage, GRP gene, Expression analysis, Biotic and abiotic stress response

Posted Date: November 19th, 2019

DOI: https://doi.org/10.21203/rs.2.17461/v1

License: (9) This work is licensed under a Creative Commons Attribution 4.0 International License.

Read Full License 


\title{
Genome-wide identification and expression analysis of glycine-rich protein genes in Chinese cabbage (Brassica rapa
}

\section{L. ssp. pekinensis)}

\author{
Xiaonan Lu${ }^{1}$, Ming Gao', Yaxiong Cheng ${ }^{1}$, Meilan $\mathrm{Li}^{1}$, Xiaoyong $\mathrm{Xu}^{1} *$ \\ ${ }^{1}$ College of Horticulture, Shanxi Agricultural University; Collaborative innovation \\ center for improving quality and increasing profits of protected vegetables in Shanxi, \\ Taigu, 030801.
}

\section{Abstract}

\section{Background}

Plant Glycine-rich proteins, a superfamily with a glycine-rich domain, play an important role in various stress such as low temperature, drought, high salt, and so on. Although the research of GRP genes has been reported in many plants, the GRP gene has seldom reported in Chinese cabbage so far. Research results made a guide to further understand the function of $B r G R P$ genes in Chinese cabbage.

\section{Results}

In this study, a total of 141 glycine-rich protein genes were identified in Chinese cabbage by homology comparative analysis. A further prediction of physical and chemical characteristics revealed that $58.3 \%$ of BrGRPs were alkalines, $63.1 \%$ of BrGRPs were unstable, and $73.8 \%$ were hydrophilic. Conserved domain analysis showed that 110 BrGRPs contained 18 same conserved motifs, and could be classified into five main subclasses which the evolutionary relationship and gene structure may be conserved while the other 31 BrGRPs, including Bra014168, Bra040002, etc, may gain new functions or gradually lost gene functions according to the evolution process. These identified $B r G R P$ genes were also located in ten chromosomes and three different subgenomes of Chinese cabbage, and 101 pairs of orthologous GRP genes were found between Chinese cabbage and Arabidopsis. According to the opened transcriptome data, we found that $138 \mathrm{BrGRP}$ genes showed abnormal expression at high temperature, 108 $B r G R P$ genes showed abnormal expression at low temperature, and 74 at drought stress, 47 at soft rot stress, while only 3 and 7 genes at ozone and salt stress, respectively. Further promoter motif analysis found that a large number of stress-related cis-acting elements, such as DRE, MYC, MYB, and ABRE, were identified in their promoter regions, which were in correspondence with previous differential expression. In addition, some BrGRP genes were involved in multiple stresses suggested their broadspectrum resistance. 


\section{Conclusion}

A total of $141 G R P$ genes were identified in Chinese cabbage, which suggested their potential roles in plant stress response. But the molecular mechanisms by which $B r G R P$ genes respond and resist biotic and abiotic stress remain unclear. These results may provide an important basis for the study of their function in Chinese cabbage.

\section{Keywords}

Chinese cabbage, GRP gene, Expression analysis, Biotic and abiotic stress response

\section{Background}

Plant glycine-rich protein (GRP) is a class of proteins consisting of glycine-rich repeat sequences. The first glycine-rich cell wall protein PtGRP1 was isolated from Petunia hybrida in 1986, and similar proteins were further found in almost all organisms [1,2], and more and more GRPs have been isolated from plants such as Zea mays, Oryza sativa, Arabidopsis thaliana, and Nicotiana tabaccum [2].

Plant GRPs can be divided into five main subclasses based on their primary structure, the conserved domain, and the arrangement of glycine repeats [3, 4]. Class I subfamily contained a typical structural feature of GRP protein, of which the glycine-rich repeats mostly appear as (Gly)n-X, where $\mathrm{n}$ is generally an odd number and $\mathrm{X}$ can be any amino acid. At the same time, the $\mathrm{N}$-terminus contains or does not contain a signal peptide sequence. For example, PtGRP1 has $67 \%$ of glycine in glycine repeat (Gly) n-X [2]. Class II of GRPs are similar to Class I at the N-terminus and contained a cysteine-rich polypeptide chain at the end of C-terminal glycine repeat region, which may play an important role in the pathogen-related process, such as AtGRP3[5]. The glycine content of class III is lower than first two classes, but the structure is abundant. For example, Oleosin-GRPs have a fat conserved area and was located in an oil-rich cell structure of tapetum, which mainly play a role in stabilizing the triglyceride and phospholipid layer in the membrane structure [6, 7]; Class IV of GRPs, also named as RNA-binding proteins (RBPs), which has no signal peptide at their N-terminus. Besides the glycinerich sequences, these proteins also have some other structures, such as the RNArecognition motif (RRM), cold-shock domain(CSD), and CCHC zinc finger structure, and so on. These RNA recognition domains are usually able to recognize each other or help those proteins which do not have any RNA recognition domain to interact with their target by binding $[8,9]$. Class V of GRPs are similar to the members of class III, which the glycine repeats are arranged differently and presented with the mixed mode of (GGX)n and (GXGX)n [4].

The function of the plant GRP family is varied in plant growth and development. The GRPs of class I \& II are almost the active components of cell walls, which play a positive regulatory role in plant cell division and organ differentiation [10]. The 
AtGRPs, belong to class II, can interact with cell wall-associated receptor protein kinase AtWak1 to participate in the signal transduction, and prevent viruses from invading plants [5]. The GRPs of class III regulates pollen development and hydration while the class IV GRPs with an RNA recognition domain are involved in molecular processes such as alternative splicing or transcriptional regulation and played an important role in stomatal regulation, seed and stamen development[10, 11]. Plant GRP genes are often specifically expressed and plays a distinctive role in different development stage or in different tissues [10]. Compared with the wild type, the deletion mutant of AtGRRBP5 showed shorter roots, smaller leaves, and shorter flower axes, meanwhile overexpressed AtGR-RBP5 promoted cell elongation and tissue growth in Arabidopsis [2].

The plant GRPs are also involved in various abiotic stress such as low temperature, drought, and high salt stress, and may play an important role in resisting the adversity stress. A notable increase of $S b G R-R N P$ was also observed in S. Bicolor seedlings when subjected to $\mathrm{NaCl}$ treatments with $1 \mathrm{M}$ and $500 \mathrm{mM}$ [12]. The ryegrass $L P G R P 1$ gene was up-regulated under cold stress treatment [13]. Compared with elevated temperature $\left(32^{\circ} \mathrm{C}\right)$, the expression level of AtGRP7 gene was higher at low temperatures [14]. $N t G R P-1 a$ was up-regulated under drought stress and can be maintained for 3 to 6 days [15]. On the other side, overexpression of AtRBG2 could increase cold tolerance and showed higher germination rate under salt stress in Arabidopsis [16], Although overexpressed AtRBG7 could also increase cold tolerance and but inhibited seed germination and plant growth under drought stress [17]. Overexpressing AtRZ-1a, a zinc finger-containing GRP, displayed retarded germination and seedling growth under salt or dehydration stress conditions in transgenic Arabidopsis [18], but the loss-offunction mutants of AtRZ-1a germinated earlier and grew faster than the wild-type plants under the same conditions[19]. Ectopic expression of AtGRP2 and AtGRP7 in rice could also increase crop yield under drought stress [20]. In a word, different GRP genes may play different functions in various tissues or stress.

Although the research of GRP genes has been reported in many plants [21-24], the GRP gene has seldom reported in Chinese cabbage so far. In previous study, we found that an Oleosin-GRP gene $B c G R P 17$ showed higher expression levels in flower buds of male fertile plant than in sterile plant but the detail function is still unknown [25]. In this study, the potential GRP genes in Chinese cabbage were identified based on the existing genome database BRAD (http://brassicadb.org/brad/) [26], and their phyletic evolution, module prediction, and chromosomal localization were further performed. Moreover, the expression patterns of these $B r G R P$ genes were also detected in different tissues and various abiotic stresses based on the opened transcriptome databases, their functions, and evolutions in development and stress response were also discussed. These results made a guide to further understand the function of $B r G R P$ genes in Chinese cabbage. 


\section{Results}

\section{Identification and characterization of BrGRP Genes in Chinese Cabbage}

For identifying the $B r G R P$ genes in Chinese cabbage, we first screened the GRP genes of Arabidopsis in the TAIR and NCBI database. A total of 85 AtGRP genes were obtained, of which 26 genes had no orthologous genes, and the remaining 59 Arabidopsis thaliana genes were used to identify a total of $141 \mathrm{BrGRP}$ genes in BRAD database. Further prediction analysis revealed that protein characteristics of these $\operatorname{BrGRP}$ genes showed great variety in molecular weight, theoretical point, etc. The length of amino acids ranged from 52aa (Bra027145.1) to 2038aa (Bra028693.1), and the theoretical point ranged from 4.11 (Bra019852.1) to 12.02 (Bra009252.1), of which $58.3 \%$ BrGRPs showed a theoretical point greater over 7. More detailed information about the instability index, aliphatic index and grand average of hydropathicity were also predicted, of which $63.1 \%$ proteins showed the aliphatic index over 40 and $73.8 \%$ proteins showed a grand average of hydropathicity with negative value. All these results suggested that these BrGRPs mainly exist on the unstable alkaline hydrophilic protein (Additional file 1: Table S1).

Subcellular localization showed that most BrGRPs (102 of 141) were secreted to the extracellular, 26 BrGRPs were localized in nuclear, 7 BrGRPs were localized in mitochondria; 3 BrGRPs were localized in plasma membrane; 2 BrGRPs, Bra040170 and Bra012653, were localized in membrane-bound chloroplast; and only one BrGRP Bra005945 was localized in chloroplast (Additional file 1: Table S1). These results could provide a reference for further functional analysis of these BrGRPs in Chinese cabbage.

\section{Analysis of phylogenetic relationships, conserved domain and gene structure of BrGRP genes}

To further classified these BrGRPs, the conserved domain analysis was performed by MEME tool. A total of $110 \mathrm{BrGRP}$ genes showed similar conserved domains in protein sequence, and a total of 18 motifs were identified including 4 glycine-rich motifs (motif 5, 6, 8, 15), 6 RNA recognition motifs (motif 1, 4, 7, 9, 12, 13), 2 CCHC-Zinc finger structural motifs (motif 11, 17), 1 cold shock motif (CSD) (motif 3), 1 Oleosin lipid motif (motif 2), a signal peptide motif (motif 10), and 3 unknown structures Motif (motif 14, 16, 18) (Fig. 1c, Additional file 2: Fig S1).

According to different motifs, 110 BrGRPs were employed to construct a phylogenetic tree by $\mathrm{Nj}$ method (Fig. 1a), and can be roughly divided into five subclasses: Class I with 8 members, which exists a cysteine-rich polypeptide chain at the end of C-terminal with glycine repeat, and similar to the Class II GRP protein defined by predecessors in Arabidopsis or others [21]. Class II with 44 members, the N-terminus of this subclasses contained a typical glycine repeat structure followed by a signal peptide sequence or not, which were like to the predecessor's Class I subfamily [4]. In addition to the 
glycine-rich region, Class III with 25 members also have RNA recognition domain, cold shock domain, and CCHC-zinc finger domain structure, which like the previous Class IV, glycine-rich RNA-binding protein subfamily. The glycine content of Class IV with 17 members was lower than previous subfamily, and belong to the predecessor's Class III subfamily. the glycine content of 16 BrGRPs was the lowest, and repeated with GGX, GX (X represent any amino acid), which was coincide to the Class V subfamily (Fig. 1c). Our classifying of five subclasses in BrGRPs was highly similar to the predecessor's subfamily classification in Arabidopsis or others, the genes in the same subgroup shared a close phylogenetic relationship, high sequence similarity, and similar gene structures, reveals the evolutionary conservation of GRP gene family.

The exon-intron structure distribution of 110 GRP genomic sequences was also analyzed (Fig. 1b), and $55 \mathrm{BrGRP}$ genes showed no intron, while the other $55 \mathrm{BrGRP}$ genes contained 2 or more exons, of which Bra011396 with 21 exons was the maximum. According to the motif classification, the exon numbers of Class I BrGRP genes subfamily were the least with 1 or 2 exons, Class II subfamily showed the maximum polymorphism from 1 to 21 exons, the others Class III, IV\& V have 1-9, 1-3 and 1-14 exons, respectively.

\section{Chromosome localization and orthologous gene analysis of BrGRPs in Chinese cabbage}

To examine the chromosomal distribution of $141 \mathrm{BrGRP}$ genes, these genes were mapped onto the chromosomes and three fractionated subgenomes of Chinese cabbage based on the B. rapa genome database (chromosome v2.5). Except $6 \mathrm{BrGRP}$ genes (Bra040395, Bra034475, Bra040817, Bra040764, Bra039380, Bra040002) could not be assigned to any chromosome, the other 135 genes were successfully located on the 10 chromosomes, in which A03 chromosome was located most with 23 BrGRP genes, while A05 and A06 chromosomes were located only 7 BrGRP genes, each. Furthermore, $44 \mathrm{BrGRP}$ genes were anchored on the least fractionated (LF) subgenome, 54 genes on the medium fractionated (MF1) subgenome, and 37 genes on the Most fractionated (MF2) subgenome (Fig.2; Additional file 1: Table S2). Moreover, 101 pairs of BrGRP genes syntenic paralogs were found on different subgenomes of Chinese cabbage (Fig.3; Additional file 1: Table S3). For example, Bra031554 and Bra030674 are located in MF2 and MF1 subgenomes, respectively, while both exhibited high sequence similarities with $A T 1 G 07135$. In order to further understand the duplication of the BrGRP genes during the whole genome duplication in Chinese cabbage, the orthologous analysis of GRP homologous genes in Chinese cabbage and Arabidopsis thaliana were also compared (Fig.3 Additional file 1: Table S3). A total of 42 AtGRP genes have found the orthologous genes in Chinese cabbage and most GRP genes of Arabidopsis have 1-7 orthologous genes in Chinese cabbage. There are also 26 AtGRP genes that have no orthologous genes in Chinese cabbage. These results revealed the evolution of $B r G R P$ genes during whole-genome duplication events in Chinese cabbage 
and would provide a strong reference for discovering the functions of $B r G R P$ genes in Chinese cabbage.

\section{Expression profiling of BrGRPs in Chinese cabbage}

Based on the published transcriptomic data, the expression of these BrGRP genes was further analyzed in Chinese cabbage under different tissues and two development stages, especially in various abiotic and biotic stress, and a set of $B r G R P$ genes were identified to be abnormal expression.

\section{Expression profiling of BrGRPs in different tissue anddevelopment stage}

The expression of these $B r G R P$ genes were compared on callus, root, stem, leaf, flower, silique, rosette and folding leaves, and just 5 BrGRPs, including Bra011869, Bra010693, Bra025663, Bra030284, and Bra031210, were detected expressing in the six tissues, their expression in flowers and siliques were lower than in other tissues but without any significant differences. Compared with other tissues, Bra011869 has the lowest expression in flower organs, and the other four genes showed the lowest expression in silique (Fig. 4a; Additional file 3: Table S4).

Besides that, the expression of $15 \mathrm{BrGRP}$ genes were found in rosette and folding leaves of different stages, of which 12 genes including Bra013176, Bra008205, Bra038769, Bra020903, Bra022895, Bra024169, Bra011077, Bra010346, Bra011195, Bra000643, Bra035944, and Bra014000, showed higher expression in rosette leaves than in folding leaves, while the rest 3 genes, Bra030674, Bra030673, and Bra030284, showed lower expressed (Fig. 4b; Additional file 3: Table S5). These found suggest that most BrGRP genes may not express or showed very lower during plant development and in normal growth conditions.

\section{Expression profiling of BrGRP genes under temperature stress}

The expression of $\operatorname{BrGRP}$ genes were compared under heat stress at 0, 0.5, 1, 2, 3 and 4 hours of heat stress with $45^{\circ} \mathrm{C}$ in 'Chiifu' seedlings [27], a total of 138 differentially expressed BrGRP genes were found (Fig. 5a, Additional file 3: Table S6), which can be divided into 28 expression patterns by STEM software (Additional file 3: Table S7). Among them, six expression patterns (profile 39, 43, 44, 46, 47 and 49) and a totally of $21 \mathrm{BrGRP}$ genes $\mathrm{BrGRP}$ genes were significantly up-regulated at 5 times under heat stress. Seven expression patterns (profile 0, 1, 8, 11, 16, 21, 35) containing $B r G R P$ genes were significantly down-regulated at 5 times under heat stress. Another 15 expression patterns showed disordered fluctuating. For example, Bra036999 of profile 9 was down-regulated at $0.5,1 \mathrm{~h}$, and $2 \mathrm{~h}$ but up-regulated at $3 \mathrm{~h}$ and $4 \mathrm{~h}$. Bra038836 of profile 22 was down-regulated at $0.5 \mathrm{~h}, 1 \mathrm{~h}$, while up-regulated at $2 \mathrm{~h}, 3 \mathrm{~h}$, and $4 \mathrm{~h}$. 
Bra025622 of profile 34 was down-regulated at $1 \mathrm{~h}, 4 \mathrm{~h}$, while up-regulated at $0.5 \mathrm{~h}, 2 \mathrm{~h}$ and $3 \mathrm{~h}$.

On the other side, a totally of $108 \mathrm{BrGRP}$ genes were found to be expression in two true leaves under cold treatment based on the transcriptome data of 29-day leaves treated with $4{ }^{\circ} \mathrm{C}$ temperature [28]. Among them, the expression of 48 genes showed significant differences in low-temperature treatment than the control with $25{ }^{\circ} \mathrm{C}$ [ $\log 2$ fold-change(FC)>1], of which 24 genes were up-regulated, and the remain 24 genes were down-regulated after low-temperature treatment (Fig. 5b, Additional file 3: Table S8).

\section{Expression profiling of BrGRPs under Drought Stress.}

To study the expression pattern of $B r G R P$ genes under drought stress, the transcriptome data of Chinese cabbage (CR2355 and ATC92037) were also analyzed [29]. CR2355 is a root transcriptome data of drought-tolerant (DT) material which can keep the required biomass for mature when suffered from transient drought stress during the reproductive phase, while ATC92037 is a root transcriptome data of drought-sensitive (DS) material which showed a significant reduction in biomass after transient drought stress. After simulant drought treatment with 2.5\% PEG 6000 in seedling, 74 BrGRP genes showed differential expression at $4 \mathrm{~h}, 8 \mathrm{~h}$, and $12 \mathrm{~h}$ between DT and DS materials, and can be further divided into 13 and 15 profile, respectively (Fig. 6, Additional file 3: Table S9, S10) Among them, some genes showed common expression pattern between DS and DT materials, for example, 8 BrGRP genes (Bra000643, Bra011869, Bra013176, Bra013176, Bra015926, Bra021807, Bra025663, Bra031210, Bra035944), belong to the similar expression patterns (profile 5,6), and showed continuous downregulated at the first $8 \mathrm{~h}$ of drought stress, and then up-regulated at $12 \mathrm{~h}$, while $12 \mathrm{BrGRP}$ genes including Bra031554, Bra032933, Bra029489, Bra020903, Bra010730, Bra037035, Bra011396, Bra030284, Bra040170, Bra012653, Bra037697, and Bra022938 were up-regulated in at least one material at $4 \mathrm{~h}$ or $8 \mathrm{~h}$ after drought treatment, and then down-regulated or returned to a normal level. There are also some BrGRP genes which showed differentiated expression between DT and DS materials, such as 10 BrGRP genes (Bra015350, Bra025674, Bra005798, Bra001972, Bra022895, Bra011869, Bra010693, Bra004190, Bra014000, Bra013997) was down-regulated or the expression level was not significantly changed in DS material, but up-regulated in DT material after drought treatment, 2 genes, Bra011196\&Bra030325, were upregulated in DS plants but down-regulated or without any changed in DT plants after drought treatment. The different expression patterns of these BrGRPs in two materials under drought stress may play different roles in the response of drought tolerance in Chinese cabbage.

\section{Expression profiling of BrGRPs under soft rot stress}

We also screened the different expression $B r G R P$ genes under soft rot stress based on the transcriptome data of soft rot-resistant mutant(sr) and wild control inoculated with soft rot at $0 \mathrm{~h}, 6 \mathrm{~h}, 12 \mathrm{~h}$ and $24 \mathrm{~h}$ [30], and totally 47 differentially expressed BrGRP genes were found (Fig. 7a, Additional file 3: Table S11), which can be divided into 20 
expression patterns (Additional file 3: Table S12). The results showed that many $B r G R P$ genes were down-regulated within 12 hours after inoculation and up-regulated at later $12 \mathrm{~h}-24 \mathrm{~h}$. while $8 \mathrm{BrGRP}$ genes were up-regulated at four times after inoculated with soft rot in mutants. These results suggested a special stress reaction of $B r G R P$ genes against pathogens.

At last, a Venn diagram simply revealed that the numbers of BrGRP genes involved in various stress (Fig. 7b, Additional file 4: Table S13). 32 BrGRP genes such as Bra033973, Bra011869, Bra035917, showed different expression under four types of stress. 39 BrGRP genes, including Bra031554, Bra037697, Bra010693, showed different expression under heat, cold and drought stress. BrGRP genes showed different expression under heat and cold stress, such as Bra013640, Bra027092.11 BrGRP genes showed different expression under heat, cold and soft rot stress, such as Bra035778, Bra040291, Bra040395, and etc. Furthermore, some BrGRP genes just differently expressed under single stress. For example, $33 \mathrm{BrGRP}$ genes are only differently expressed under heat stress while just Bra000790 showed different expression under cold stress.

At the same time, we also found that the expression pattern of $B r G R P$ genes under different abiotic stresses is not completely consistent. For example, Bra030284 is upregulated under both high and low temperature stress, while down-regulated under drought stress, and also showed up-regulated expression in drought-stressed DS plants and down-regulation in DT plants. Bra010693 was up-regulated under low temperature stress and in drought-stressed DT plants, while down-regulated under high temperature stress and in drought-stressed DS plants. Bra011869 was down-regulated under low temperature, high temperature, and in drought stressed DS material, while up-regulated under drought stressed DT material and in soft rot stress. The different expression of these $B r G R P$ genes under various tissues and in multiple stresses may due to the different functional dissimilation, which need further certificated.

\section{Analysis of cis-acting elements in the promoter region of BrGRPs}

In order to declare the molecular mechanism of abnormally expressed $B r G R P$ genes under various adversity stresses, $141 \mathrm{BrGRP}$ genes are classified according to the types of biotic and abiotic stresses in the Venn diagram and the stress-related cis-acting elements of their promoter regions were further analyzed (Fig 8, Additional file5: Table S14). The results indicate that lots of stress-related cis-acting elements were found in the promoter regions of $141 \mathrm{BrGRP}$ genes, including DRE, MYB, MYC, ABRE, LTRE, W-box, TC-rich repeats, and so on. DRE has been identified as a cis-acting element involved in drought, high salt and low-temperature stress, MYB involved in by drought, cold and salt stress [31], MYC involved in drought and ABA [32], ABRE involved in ABA and drought stress [33], LTRE involved in low temperature stress [34]. W-box binds to WRKY transcription factor, participates in the stress response of plants such 
as diseases, drought, $\mathrm{ABA}$, and etc [35]. TC-rich repeats participates in plant defense and stress response[36].

The number of stress-related cis-acting elements in the promoter region of these 141 $B r G R P$ genes were different, from the minimum with 4 (Bra032933) to the maximum with 34 (Bra009254), and each promoter region of $B r G R P$ genes distributed 3-6 types of cis-acting elements. 97.2\% (137/141) of BrGRP genes promoter region contained MYC element, 98.6\% (139/141) contained MYB element, 84.4\% (119/141) has ABRE element, 55.3\% (78/141) has LTRE element, 26.2\% (37/141), 51.1\%(72/141) and 40.4\%(57/141) contained DRE, W-box, and TC-rich repeats, respectively. These cisacting elements distributed in the promoter region of $B r G R P$ genes played an important role in the response of adversity stress which further verified the expression difference identified previously in Chinese cabbage. There are also different types of stress-related cis-acting elements in the promoter region of the BrGRP genes. For example, Bra037697, Bra037056, and other 15 genes only have ABRE, LTRE, MYB, and MYC. Bra030582, Bra032844, and other 9 genes only have ABRE, LTRE, MYB, MYC and TC rich repeats. Differences in the type of cis-acting elements of the BrGRP genes promoter region may be associated with different expression patterns of different $\operatorname{BrGRP}$ genes under biotic and abiotic stress. At the same time, the number of stress response cis-acting elements in the promoter region is not completely consistent with the expression pattern under the stress of BrGRP genes. For example, the ABRE element related to drought [33] is not found in the promoter region of Bra000790 with a specific response under low-temperature stress. 11 ABRE elements were found in the promoter region of Bra028691 who is specific response for high-temperature stress. This may be due to the loss of related functions by missing some of the cis-acting elements in promoter region. These results may provided an important reference for exploring the action mechanism of $B r G R P$ genes in biotic and abiotic resistance.

\section{Discussion}

The plant GRP gene family is a superfamily with the domain of glycine repeat (Gly) n$X$. However, due to the diversification of their protein domains, gene expression patterns, and subcellular localization, these GRP genes sometimes are also not considered to be a gene superfamily but a group of proteins with some repeating structural motifs [21], which may disturb the identification of GRP gene family. Anyway, 15, 22, 12, and 18 glycine-rich RNA-binding proteins (RBGs) have been identified in Arabidopsis, Chinese cabbage, rice and maize genomes, respectively [22, 24], furthermore, 9 and 51 GRP genes have been identified in sweet potato and Curcuma longa L., respectively [21, 23]. In this paper, $141 \mathrm{BrGRP}$ genes were preliminarily identified in Chinese cabbage based on the BRAD genome sequence. Except 31 genes without any conserved domain which may due to the gradual loss of functions or get the new function during evolution [37], the remaining $110 \mathrm{BrGRP}$ genes could be roughly divided into five main subclasses according to the conserved domain, which was similar to the previous studies $[3,4]$. The numbers of glycine repeat 
in the glycine-rich region of BrGRPs were always as even and usually exist with the order of (Gly)6-X (motif 6), that was different from the other plants which contained the odd numbers $[3,4]$. This different arrangement of BrGRP genes may lead to the unique function in Chinese cabbage which needs further study.

Among five main subfamilies, the BrGRPs of class I and class II in Chinese cabbage are similar in the term of the conserved domain, glycine repeat motif, evolutionary phylogeny and gene structure (1-3 exons), except a cysteine-rich region at the Cterminus of class II. Simultaneously, the Class IV and Class V proteins are also similar except the conserved Oleosin domain in Class IV. These results suggested that the BrGRPs of class I \& class II, Class IV \& Class V, each have the same ancestors which may develop additional conserved domain and get new functions during the evolution process. In addition, we also identified three Chinese cabbage Zinc Finger-Containing Glycine-Rich RNA-Binding Proteins（RZs） [38] from the Class III proteins, including Bra032933, Bra025250, Bra005798, all of which contained three single structural domains RRM, CSD, and CCHC, their function is still unknown.

Chinese cabbage genome was not only suffered the evolution events of three-time genome-wide replication shared with other cruciferous plants[39, 40], but also divided into three subgenomic groups, named as LF, MF1, MF2 subgenome according to the number of gene losses from small to large $[37,41]$. In this paper, the syntenic analysis of GRP genes between Chinese cabbage and Arabidopsis thaliana also verified this genome-wide replication event that Chinese cabbage was originated from the hexaploid ancestor, and was rearranged to be diploid as the current cabbage species after chromosome fusion [41]. Meanwhile, it was found that $141 \mathrm{BrGRP}$ genes of Chinese cabbage, including 44 LF genes, 54 MF1 genes, and 37 MF2 genes, were not in a threefold relationship with 85 GRP genes of Arabidopsis, which may suggest that BrGRP genes of Chinese cabbage still evolved after the genome-wide replication event, and occurred larger-scale loss-of-function to prevent functional redundancy.

The expression of GRP genes in Arabidopsis thaliana always showed a tissue or organspecific manner, GRP gene of class I\&II were mainly expressed in seeds, siliques, roots, and leaves; Class III has the highest expression in shoot tips, rosettes, seeds, and flowers; Class IV is highly expressed in seeds, siliques, rosettes, and flowers; Class V is upregulated only in inflorescence [10, 42]. Although differential expression of $B r G R P$ genes were also found in different tissues in Chinese cabbage [43], the related genes were infrequent, and only part of class III genes showed different expression between various tissue. The class I and II of GRP genes may be acted as the active components of plant cell walls and played a crucial role in plant cell growth and organ differentiation [5]. The class III of BrGRP genes has strong RNA recognition and binding ability and may participate in the molecular process of plant growth and development by activating splices or regulating transcription [10]. In the study, we found that the BrGRP genes that differentially expressed at various developmental stages of Chinese cabbage belonged to the class I-III, which was similar to the previous studies, and may contain 
similar functions in terms of growth and development. Besides that, we also found that only a small number of $B r G R P$ genes showed different expression in various tissues and organs of Chinese cabbage, while most BrGRP genes expression can be induced by biotic and abiotic stress.

The plant GRP genes which can be induced by various stresses suggested another role in plant resistance. Eight glycine-rich RNA-binding protein genes (AtGR-RBP1 - AtGR$R B P 8$ ) have been reported in Arabidopsis thaliana. except AtGR-RBP5 and AtGR$R B P 6$, another $6 A t G R-R B P$ genes were strongly induced by low-temperature stress [18, 44]. Under drought and salt stress, the expression of $A t G R-R B P 1$ gene was increased but the expression of $A t G R-R B P 4$ and $A t G R-R B P 7$ were gradually attenuated, while the expression of AtGR-RBP5 and AtGR-RBP6 did not change [44]. In tobacco, the expression of $N t G R P 1$ gene was induced and continuously increased at the first 24 hours of waterlogging stress, and then decreased, while kept in a low-level under whatever high or low temperature, drought, high salt and ABA stress [15, 45]. NtRGP2 and NtRGP3 were also induced by waterlogging, high and low-temperature stress, but were not affected by ABA treatment with $100 \mu \mathrm{mol}$. L-1 [15]. Among the four glycinerich RNA-binding protein genes (OsGR-RBP1-OsGR-RBP4) in rice, only OsGR-RBP4 was induced by high temperature, high salt and drought stress [46]. In this study, a total of $141 \mathrm{BrGRP}$ genes were identified which may different expressions under high \& low temperature, drought, or soft rot stress. Although the $B r G R P$ genes that expressed in different biotic and abiotic stresses were different, and a total of 138, 108, 74 and 47 of $\operatorname{BrGRP}$ genes were expressed under high temperature, low temperature, drought, and soft rot treatment, respectively, the same $B r G R P$ gene that differentially expressed, also showed the different expression dynamics under various stresses, suggested their multiple expression patterns and different functions under adversity stress in Chinese cabbage. AtRZ-1 was strongly induced by low temperature and freezing stress, but had a negative regulation on seed germination and seedling growth under drought and high salt stress [18, 19, 47], Overexpression of AtRZ-1B and AtRZ-1C or loss of function mutations does not affect Arabidopsis seed germination and seedling growth under the same stress conditions [48]. AtRZ-1B/1C in regulating RNA splicing, gene expression, and many key aspects of plant development via interaction with proteins including SR proteins [49]. All these suggested that the $B r G R P$ gene not only responds to the multiple stresses, but also resists to the various stress by different molecular mechanisms.

A large number of stress-related elements identified in the promoter region of $B r G R P$ genes also proved an inextricable link and different response mechanisms between BrGRP genes and abiotic stress. Six important stress-related cis-acting elements of MYC, MYB, ABRE, LTRE, DRE and TC rich repeats were identified in the promoter region of $B r G R P$ genes. Multiple types of cis-acting elements lied in the promoter region may be one of the reasons for the diverse expression patterns of $B r G R P$ genes. Compared with the special expression patterns of $B r G R P$ genes under stress, $B r G R P$ genes that involved in various stresses, may play important role in broad-spectrum resistance, which should be worthy of deep research in the future. 


\section{Conclusion}

In this study, $141 \mathrm{BrGRP}$ genes were identified in the Chinese cabbage genome based on the 59 AtGRP genes of Arabidopsis. The BrGRP genes in Chinese cabbage are mainly composed of alkaline hydrophilic unstable protein and were secreted outside the cell membrane, only a few were found in organelles such as nuclear, mitochondria, and chloroplast. Some $B r G R P$ genes may gradually lose their conserved domain during evolution, and just 110 BrGRPs shared the same conserved domain, which can be divided into five subclasses. Chromosomal localization of these BrGRP genes and syntenic analysis with Arabidopsis thaliana strongly confirmed that Chinese cabbage did undergo a genome-wide triple duplication event during evolution. Their specific expression under various stresses were screened, and 3-6 types of response stress cisacting elements in the promoter region of these $\operatorname{BrGRP}$ genes were also identified, which suggested their potential roles in plant stress response. But the molecular mechanisms by which $B r G R P$ genes respond and resist biotic and abiotic stress remain unclear. These results may provide an important basis for the study of their function in Chinese cabbage.

\section{Methods}

\section{Identification of GRP genes in Chinese cabbage}

To identify the BrGRP genes in Chinese cabbage, the GRP genes in Arabidopsis thaliana were isolated from TAIR database (http://www.arabidopsis.org/) [50] and used as a query to search the homeotic BrGRP genes in Chinese cabbage genome database (BRAD v1.5, http://brassicadb.org/brad/)[26]. Their nucleotide and deduced amino acid sequences were downloaded for the following characterization analysis.

\section{Characterization analysis and subcellular localization prediction of BrGRPs}

physical and chemical characteristics, including the molecular weight (MW), theoretical point (pI), instability index, aliphatic index and grand average of hydropathicity (GRAVY), of these sequences of BrGRPs were further analyzed using the ProtParam tool of ExPASy (http://web.expasy.org/protparam/). The subcellular localization of these BrGRPs were predicted by the ProtComp tool in Softberry online website (http://linux1.softberry.com/).

\section{Multiple sequence alignment and phylogenetic tree establishment}

Multiple sequence alignments of the protein sequences were performed by Clustal $\mathrm{X}$ with default parameters. The phylogenetic tree was constructed by Neighbor-Joining method (NJ) in software MEGA-X[51], in which the check parameter bootstrap value 
was set as 1000 times, the genetic distance calculation model was set as P-distance, and the processing of vacant missing data is set as Pairwise deletion.

\section{Analysis of the conserved domain and gene structure of $B r G R P$ genes}

The structures of the coding/non-coding region of BrGRP genes were mapped by software TBtools [52]. Next, the conservative motifs were analyzed by MEME tool (version 5.0.3; http://meme-suite.org/tools/meme) with the number of motifs set as 20 and the other parameters were default values. The LOGO of conservative motifs was listed, and the corresponding Scalable Vector Graphics (SVGs) was also exported by TBtools [52].

\section{Identification of the orthologous BrGRP genes and syntenic analysis in Chinese cabbage}

According to genomic and chromosome database (v2.5) of Chinese cabbage[26, 37], the identified $\operatorname{BrGRP}$ genes were located into 10 chromosomes was and three fractionated subgenomes and the chromosome positions of $\operatorname{BrGRP}$ gene was constructed by MapChart software [53]. The syntenic analysis of BrGRP homologs between Chinese cabbage and Arabidopsis thaliana were searched by Chinese cabbage genome database (BRAD v1.5, http://brassicadb.org/brad/) [26] and the corresponding circos were drawn out by TBtools [52].

\section{Expression pattern analysis of BrGRP genes}

To analyze the expression pattern of these BrGRP genes in Chinese cabbage, the transcriptome data of B. rapa 'Chiifu' [43] and the inbred line 'Fushanbaotou', a typical heading Chinese cabbage [54] were downloaded and used for gene expression profiling in eight tissues including callus, root, stem, leaf, flower, silique, rosette and folding leaves.

The expression differences of BrGRP genes under biotic and abiotic stress were also analyzed based on the transcriptome data of Chinese cabbage under high temperature at $45^{\circ} \mathrm{C}$ [27], low temperature at $4{ }^{\circ} \mathrm{C}$ [28], drought [29], soft rot stress [30]. The expression levels of $B r G R P$ genes were calculated with the Fragments Per kb per Million reads (FPKM) values [55] and displayed with cluster heat map drawn by TBtools [52]. Analysis of expression pattern of BrGRP gene under high temperature, drought and soft rot stress by STEM (Short Short Time-series Expression Miner) [56]. The Venn map was generated by TBtools [52] according to the differentially expressed data.

\section{Analysis of the cis-acting elements in the promoter region of BrGRP genes}

To further identified the cis-acting elements in the promoter regions of $B r G R P$ genes, a $2 \mathrm{~kb}$ fragment in upstream of the ATG (start codon) were extracted by TBtools[52] and were further identified by PlantCare (http://bioinformatics.psb.ugent.be/webtools/plantcare/html/) with the adversity 
related cis-acting element including MYC (CANNTG), MYB (C/TAACNA/G), ABRE (ABA-responsive element, ACGT), LTR (low-temperature-responsive element, CCGAAA), DRE (CCGAC), W box(TTGACC) and TC rich repeats(GTTTTCTTAC). Constructing a Venn diagram by TBtools[52] based on the type of cis-acting element.

\section{List of abbreviations}

GRP: glycine-rich protein; RBPs: RNA-binding proteins; BRAD: Brassica database; TAIR: The Arabidopsis Information Resource; BrGRP: glycine-rich proteins in Chinese cabbage; RZs: Zinc Finger-Containing Glycine-Rich RNA-Binding Proteins; LF: The least fractionatedsubgenome; MF1: The medium fractionated subgenome; MF2: The mostfractionated subgenome; FPKM: Fragments Per kb per Million reads; ABA: Abscisic acid; NJ: Neighbourhood-joining method; Scalable SVGs: Vector Graphics.

\section{Ethics approval and consent to participate}

Not applicable

\section{Consent for publication}

Not applicable

\section{Availability of data and materials}

\section{Competing interests}

The authors declare that they have no competing interests

\section{Acknowledgments}

\section{Funding}

This research was supported by grants from the National Natural Science Foundation of China (31401885); the Natural Science Foundation of Shanxi Province (201801D121248); and by Shanxi Province Key Research and Development Program Key Projects (201703D211001-04-01).

\section{Author information}

\section{contributions}

$\mathrm{XL}$ and $\mathrm{XX}$ conceived \& designed the experiments. XL and $\mathrm{YC}$ wrote the manuscript. $\mathrm{XL}, \mathrm{YC}$ and $\mathrm{MG}$ were responsible for data analysis and revising the manuscript. All authors read and approved the final manuscript. ML provided helpful advice on data analysis. ML and XX revised the paper and supervised the research.

\section{*Corresponding authors:}

Xiaoyong Xu xuxy7926@163.com 


\section{References}

3 1. Condit CM, Meagher RB: A gene encoding a novel glycine-rich structural protein of petunia. Nature 1986, 323(6084):178-181;doi:10.1038/323178a0.

2. Mangeon A, Magioli C, Menezes-Salgueiro AD, Cardeal V, de Oliveira C, Galvao VC, Margis R, Engler G, Sachetto-Martins G: AtGRP5, a vacuole-located glycine-rich protein involved in cell elongation. Planta 2009, 230(2):253-265; doi:10.1007/s00425-009-0940-4.

3. Fusaro, Adriana, Mangeon, Amanda, Junqueira, Magrani R, Rocha, Ben“āacio CAa, Coutinho, Cardoso $\mathrm{T}$ et al: Classification, expression pattern and comparative analysis of sugarcane expressed sequences tags (ESTs) encoding glycine-rich proteins (GRPs). Genetics and Molecular Biology 2001;doi:10.1590/s1415-47572001000100035.

4. Bocca SN, Magioli C, Mangeon A, Junqueira RM, Cardeal V, Margis R, Sachetto-Martins G: Survey of glycine-rich proteins (GRPs) in the Eucalyptus expressed sequence tag database (ForEST). Genetics and Molecular Biology 2005, 28:608-624;doi:10.1590/S141547572005000400016.

5. Park AR, Cho SK, Yun UJ, Jin MY, Lee SH, Sachetto-Martins G, Park OK: Interaction of the Arabidopsis receptor protein kinase Wak1 with a glycine-rich protein, AtGRP-3. J Biol Chem 2001, 276(28):26688-26693;doi:10.1074/jbc.M101283200.

6. Ferreira MA, Engler JDA, Miguens FC, Montagu MV, Engler G, Oliveira DED: Oleosin gene expression in Arabidopsis thaliana tapetum coincides with accumulation of lipids in plastids and cytoplasmic bodies. Plant Physiology \& Biochemistry 1997, 35(9):729739;doi:10.1104/pp.115.1.283.

7. Murphy DJ, Hernández-Pinzón I, Patel K: Role of lipid bodies and lipid-body proteins in seeds and other tissues. Journal of Plant Physiology 2001, 158(4):471-478;doi:10.1078/0176-161700359.

8. Dreyfuss G, Kim VN, Kataoka N: Messenger-RNA-binding proteins and the messages they carry. Nat Rev Mol Cell Biol 2002, 3(3):195-205; doi:10.1038/nrm760.

9. Lorkovic ZJ, Barta A: Genome analysis: RNA recognition motif (RRM) and $\mathrm{K}$ homology (KH) domain RNA-binding proteins from the flowering plant Arabidopsis thaliana. Nucleic Acids Res 2002, 30(3):623-635; doi:10.1093/nar/30.3.623.

10. Czolpinska M, Rurek M: Plant Glycine-Rich Proteins in Stress Response: An Emerging, Still Prospective Story. Front Plant Sci 2018, 9:302;doi:10.3389/fpls.2018.00302.

11. Winter D, Vinegar B, Nahal H, Ammar R, Wilson GV, Provart NJ: An "Electronic Fluorescent Pictograph" browser for exploring and analyzing large-scale biological data sets. PLoS One 2007, 2(8):e718;doi:10.1371/journal.pone.0000718.

12. Aneeta, Sanan-Mishra N, Tuteja N, Kumar Sopory S: Salinity- and ABA-induced up-regulation and light-mediated modulation of mRNA encoding glycine-rich RNA-binding protein from Sorghum bicolor. Biochem Biophys Res Commun 2002, 296(5):1063-1068;doi:10.1016/s0006291x(02)02050-8.

13. Shinozuka H, Hisano H, Yoneyama S, Shimamoto $Y$, Jones ES, Forster JW, Yamada T, Kanazawa A: Gene expression and genetic mapping analyses of a perennial ryegrass glycine-rich RNAbinding protein gene suggest a role in cold adaptation. Mol Genet Genomics 2006, 275(4):399408;doi:10.1007/s00438-005-0095-3. 
14. Wienkoop S, Morgenthal K, Wolschin F, Scholz M, Selbig J, Weckwerth W: Integration of metabolomic and proteomic phenotypes: analysis of data covariance dissects starch and RFO metabolism from low and high temperature compensation response in Arabidopsis thaliana. Mol Cell Proteomics 2008, 7(9):1725-1736;doi:10.1074/mcp.M700273-MCP200.

15. Chen XA, Zeng QC, Lu XP, Yu DQ, Li WZ: Characterization and Expression Analysis of Four Glycine-Rich RNA-Binding Proteins Involved in Osmotic Response in Tobacco (Nicotiana tabacum cv. Xanthi). Agricultural Sciences in China 2010, 9(11):1577-1587;doi:10.1016/S16712927(09)60254-6.

16. Kim JY, Park SJ, Jang B, Jung CH, Ahn SJ, Goh CH, Cho K, Han O, Kang H: Functional characterization of a glycine-rich RNA-binding protein $\mathbf{2}$ in Arabidopsis thaliana under abiotic stress conditions. Plant J 2007, 50(3):439-451;doi:10.1111/j.1365-313X.2007.03057.x.

17. Kim JS, Jung HJ, Lee HJ, Kim KA, Goh CH, Woo Y, Oh SH, Han YS, Kang H: Glycine-rich RNAbinding protein 7 affects abiotic stress responses by regulating stomata opening and closing in Arabidopsis thaliana. Plant $J$ 2008, 55(3):455-466; doi:10.1111/j.1365-313X.2008.03518.x.

18. Kim YO, Kim JS, Kang $\mathrm{H}$ : Cold-inducible zinc finger-containing glycine-rich RNA-binding protein contributes to the enhancement of freezing tolerance in Arabidopsis thaliana. Plant J 2005, 42(6):890-900;doi:10.1111/j.1365-313X.2005.02420.x.

19. Kim YO, Pan $\mathrm{S}$, Jung $\mathrm{CH}$, Kang $\mathrm{H}$ : A zinc finger-containing glycine-rich RNA-binding protein, atRZ-1a, has a negative impact on seed germination and seedling growth of Arabidopsis thaliana under salt or drought stress conditions. Plant Cell Physiol 2007, 48(8):11701181;doi:10.1093/pcp/pcm087.

20. Yang DH, Kwak KJ, Kim MK, Park SJ, Yang KY, Kang H: Expression of Arabidopsis glycine-rich RNA-binding protein AtGRP2 or AtGRP7 improves grain yield of rice (Oryza sativa) under drought stress conditions. Plant Sci 2014, 214:106-112;doi:10.1016/j.plantsci.2013.10.006.

21. Kar B, Nayak S, Joshi RK: Classification and comparative analysis of Curcuma longa L. expressed sequences tags (ESTs) encoding glycine-rich proteins (GRPs). Bioinformation 2012, 8(3):142-146; doi:10.6026/97320630008142.

22. Krishnamurthy $\mathrm{P}$, Kim JA, Jeong M, Kang $\mathrm{CH}$, Lee SI: Defining the RNA-binding glycine-rich (RBG) gene superfamily: new insights into nomenclature, phylogeny, and evolutionary trends obtained by genome-wide comparative analysis of Arabidopsis, Chinese cabbage, rice and maize genomes. Molecular Genetics and Genomics 2015, 290(6):22792295; doi:10.1007/s00438-015-1080-0.

23. Lu Y, Sun J, Yang Z, Zhao C, Zhu M, Ma D, Dong T, Zhou Z, Liu M, Yang D et al: Genome-wide identification and expression analysis of glycine-rich RNA-binding protein family in sweet potato wild relative Ipomoea trifida. Gene 2019, 686:177186; doi:10.1016/j.gene.2018.11.044.

24. Zhang J, Zhao Y, Xiao H, Zheng Y, Yue B: Genome-wide identification, evolution, and expression analysis of RNA-binding glycine-rich protein family in maize. J Integr Plant Biol 2014, 56(10):1020-1031;doi:10.1111/jipb.12210.

25. $\mathrm{Xu} X Y$, Sun $X L$, Zhang J, Huang $W W$, Zhang LG, Fang $Z Y$ : Identification of candidate genes associated with male sterility in CMS7311 of heading Chinese cabbage (Brassica campestris L. ssp pekinensis). Acta Physiol Plant 2013, 35(11):3265-3270

26. Cheng F, Liu S, Wu J, Fang L, Sun S, Liu B, Li P, Hua W, Wang X: BRAD, the genetics and genomics database for Brassica plants. BMC Plant Biol 2011, 11:136;doi:10.1186/1471-2229-11-136. 
27. Dong X, Yi H, Lee J, Nou IS, Han CT, Hur Y: Global Gene-Expression Analysis to Identify Differentially Expressed Genes Critical for the Heat Stress Response in Brassica rapa. PLoS One 2015, 10(6):e0130451;doi:10.1371/journal.pone.0130451.

28. Zhang CW, Wei YP, Xiao D, Gao LW, Lyu SW, Hou XL, Bouuema G: Transcriptomic and proteomic analyses provide new insights into the regulation mechanism of low-temperature-induced leafy head formation in Chinese cabbage. J Proteomics 2016, 144:110;doi:10.1016/j.jprot.2016.05.022.

29. Guo YM, Samans B, Chen S, Kibret KB, Hatzig S, Turner NC, Nelson MN, Cowling WA, Snowdon RJ: Drought-Tolerant Brassica rapa Shows Rapid Expression of Gene Networks for General Stress Responses and Programmed Cell Death Under Simulated Drought Stress. Plant Mol Biol Report 2017, 35(4):416-430;doi:10.1007/s11105-017-1032-4.

30. Liu M, Wu F, Wang S, Lu Y, Chen X, Wang Y, Gu A, Zhao J, Shen S: Comparative transcriptome analysis reveals defense responses against soft rot in Chinese cabbage. Hortic Res 2019, 6:68;doi:10.1038/s41438-019-0149-z.

31. Dai X, Xu Y, Ma Q, Xu W, Wang T, Xue Y, Chong K: Overexpression of an R1R2R3 MYB gene, OsMYB3R-2, increases tolerance to freezing, drought, and salt stress in transgenic Arabidopsis. Plant Physiol 2007, 143(4):1739-1751;doi:10.1104/pp.106.094532.

32. Onishi M, Tachi H, Kojima T, Shiraiwa M, Takahara H: Molecular cloning and characterization of a novel salt-inducible gene encoding an acidic isoform of PR-5 protein in soybean (Glycine $\max$ [L.] Merr.). Plant Physiol Biochem 2006, 44(10):574580;doi:10.1016/j.plaphy.2006.09.009.

33. Manavella PA, Dezar CA, Ariel FD, Chan RL: Two ABREs, two redundant root-specific and one W-box cis-acting elements are functional in the sunflower HAHB4 promoter. Plant Physiol Biochem 2008, 46(10):860-867; doi:10.1016/j.plaphy.2008.05.003.

34. Maestrini P, Cavallini A, Rizzo M, Giordani T, Bernardi R, Durante M, Natali L: Isolation and expression analysis of low temperature-induced genes in white poplar (Populus alba). J Plant Physiol 2009, 166(14):1544-1556;doi:10.1016/j.jplph.2009.03.014.

35. Wang Z, Zhu Y, Wang L, Liu X, Liu Y, Phillips J, Deng X: A WRKY transcription factor participates in dehydration tolerance in Boea hygrometrica by binding to the $\mathbf{W}$-box elements of the galactinol synthase (BhGolS1) promoter. Planta 2009, 230(6):1155-1166;doi:10.1007/s00425009-1014-3.

36. Banerjee J, Sahoo DK, Dey N, Houtz RL, Maiti IB: An intergenic region shared by At4g35985 and At4g35987 in Arabidopsis thaliana is a tissue specific and stress inducible bidirectional promoter analyzed in transgenic arabidopsis and tobacco plants. PLOS One 2013, 8(11):e79622;doi:10.1371/journal.pone.0079622.

37. Cheng F, Mandakova T, Wu J, Xie Q, Lysak MA, Wang X: Deciphering the diploid ancestral genome of the Mesohexaploid Brassica rapa. Plant Cell 2013, 25(5):15411554;doi:10.1105/tpc.113.110486.

38. $\mathrm{Xu} \mathrm{T,} \mathrm{Gu} \mathrm{L,} \mathrm{Choi} \mathrm{MJ,} \mathrm{Kim} \mathrm{RJ,} \mathrm{Suh} \mathrm{MC,} \mathrm{Kang} \mathrm{H:} \mathrm{Comparative} \mathrm{Functional} \mathrm{Analysis} \mathrm{of} \mathrm{Wheat}$ (Triticum aestivum) Zinc Finger-Containing Glycine-Rich RNA-Binding Proteins in Response to Abiotic Stresses. Plos One 2014, 9(5);doi:ARTN e96877 10.1371/journal.pone.0096877.

39. Thomas BC, Pedersen B, Freeling M: Following tetraploidy in an Arabidopsis ancestor, genes were removed preferentially from one homeolog leaving clusters enriched in dose-sensitive genes. Genome Res 2006, 16(7):934-946;doi:10.1101/gr.4708406. 
40. Franzke A, Lysak MA, Al-Shehbaz IA, Koch MA, Mummenhoff K: Cabbage family affairs: the evolutionary history of Brassicaceae. Trends Plant Sci 2011, 16(2):108116;doi:10.1016/j.tplants.2010.11.005.

41. Wang $X$, Wang $H$, Wang J, Sun R, Wu J, Liu S, Bai Y, Mun JH, Bancroft I, Cheng F et al: The genome of the mesopolyploid crop species Brassica rapa. Nat Genet 2011, 43(10):10351039; doi:10.1038/ng.919.

42. Vivek K, Hanlon MR, Sergio C, Ferlanti ES, Svetlana K, Maria K, Rosen BD, Chia-Yi C, Walter M, Mock SA: Araport: the Arabidopsis information portal. Nucleic Acids Research 2015, 43(Database issue):1003-1009

43. Tong C, Wang X, Yu J, Wu J, Li W, Huang J, Dong C, Hua W, Liu S: Comprehensive analysis of RNA-seq data reveals the complexity of the transcriptome in Brassica rapa. BMC Genomics 2013, 14:689;doi:10.1186/1471-2164-14-689.

44. Kwak KJ, Kim YO, Kang H: Characterization of transgenic Arabidopsis plants overexpressing GR-RBP4 under high salinity, dehydration, or cold stress. J Exp Bot 2005, 56(421):30073016; doi:10.1093/jxb/eri298.

45. Lee MO, Kim KP, Kim BG, Hahn JS, Hong CB: Flooding stress-induced glycine-rich RNA-binding protein from Nicotiana tabacum. Mol Cells 2009, 27(1):47-54;doi:10.1007/s10059-009-00044.

46. Sahi $C$, Agarwal $M$, Singh A, Grover A: Molecular characterization of a novel isoform of rice ( Oryza sativa L.) glycine rich-RNA binding protein and evidence for its involvement in high temperature stress response. Plant Science 2007, 173(2):144155;doi:10.1016/j.plantsci.2007.04.010.

47. Kim $\mathrm{YO}$, Kang $\mathrm{H}$ : The role of a zinc finger-containing glycine-rich RNA-binding protein during the cold adaptation process in Arabidopsis thaliana. Plant Cell Physiol 2006, 47(6):793798; doi:10.1093/pcp/pcj047.

48. Kim WY, Kim JY, Jung HJ, Oh SH, Han YS, Kang H: Comparative analysis of Arabidopsis zinc finger-containing glycine-rich RNA-binding proteins during cold adaptation. Plant physiology and biochemistry : PPB 2010, 48(10-11):866-872;doi:10.1016/j.plaphy.2010.08.013.

49. Wu Z, Zhu DL, Lin XY, Miao J, Gu LF, Deng X, Yang Q, Sun KT, Zhu DM, Cao XF et al: RNA Binding Proteins RZ-1B and RZ-1C Play Critical Roles in Regulating Pre-mRNA Splicing and Gene Expression during Development in Arabidopsis. Plant Cell 2016, 28(1):5573;doi:10.1105/tpc.15.00949.

50. Lamesch P, Berardini TZ, Li D, Swarbreck D, Wilks C, Sasidharan R, Muller R, Dreher K, Alexander DL, Garcia-Hernandez M et al: The Arabidopsis Information Resource (TAIR): improved gene annotation and new tools. Nucleic Acids Res 2012, 40(Database issue):D12021210;doi:10.1093/nar/gkr1090.

51. Kumar S, Stecher G, Li M, Knyaz C, Tamura K: MEGA X: Molecular Evolutionary Genetics Analysis across Computing Platforms. Mol Biol Evol 2018, 35(6):15471549;doi:10.1093/molbev/msy096.

52. Chen C, Xia R, Chen H, He Y: TBtools, a Toolkit for Biologists integrating various HTS-data handling tools with a user-friendly interface. bioRxiv 2018:289660;doi:10.1101/289660.

53. Voorrips RE: MapChart: software for the graphical presentation of linkage maps and QTLs. J Hered 2002, 93(1):77-78;doi:10.1093/jhered/93.1.77.

54. Wang F, Li L, Li H, Liu L, Zhang Y, Gao J, Wang X: Transcriptome analysis of rosette and folding 
leaves in Chinese cabbage using high-throughput RNA sequencing. Genomics 2012, 99(5):299-307; doi:10.1016/j.ygeno.2012.02.005.

55. Mortazavi A, Williams BA, McCue K, Schaeffer L, Wold B: Mapping and quantifying mammalian transcriptomes by RNA-Seq. Nat Methods 2008, 5(7):621-628;doi:10.1038/nmeth.1226.

56. Ernst J, Bar-Joseph Z: STEM: a tool for the analysis of short time series gene expression data. BMC Bioinformatics 2006, 7:191;doi:10.1186/1471-2105-7-191.

57. Lescot $M$, Dehais $P$, Thijs G, Marchal K, Moreau Y, Van de Peer $Y$, Rouze P, Rombauts S: PlantCARE, a database of plant cis-acting regulatory elements and a portal to tools for in silico analysis of promoter sequences. Nucleic Acids Res 2002, 30(1):325327;doi:10.1093/nar/30.1.325.

\section{Additional files}

Additional file1 Table S1 The information on BrGRP genes in Chinese cabbage. Additional file1 Table S2 The distribution of BrGRP genes in genome of Chinese cabbage.

Additional file1 Table S3 The orthologous gene pairs in GRP genes of Chinese cabbage and Arabidopsis thaliana.

Additional file 2 Figure S1 The LOGO of GRP conservative motifs in Chinese cabbage.

Additional file3 Table S4 The FPKM values of $B r G R P$ genes in five different tissues of Chinese cabbage.

Additional file3 Table S5 The expression values of $B r G R P$ genes in defferent development stages of Chinese cabbage.

Additional file3 Table S6 The expression values of $B r G R P$ genes under $45{ }^{\circ} \mathrm{C}$ heat treatment in Chinese cabbage.

Additional file3 Table S7 The expression profiles of $B r G R P$ genes under $45{ }^{\circ} \mathrm{C}$ heat treatment in Chinese cabbage.

Additional file3 Table S8 The expression values of $B r G R P$ genes under low temperature treatment in Chinese cabbage.

Additional file3 Table S9 The expression values of $B r G R P$ genes under drought treatment in Chinese cabbage.

Additional file3 Table S10 The expression profiles of $B r G R P$ genes under drought treatment in Chinese cabbage.

Additional file3 Table S11 The expression values of BrGRP genes under soft rot treatment in Chinese cabbage.

Additional file3 Table S12 The expression profiles of BrGRP genes under soft rot treatment in Chinese cabbage.

Additional file4 Table S13 The same BrGRP genes expression under different biotic and abiotic stresses.

Additional file5 Table S14 Quantitative statistics of cis-acting elements related to adversity stress in the promoter region of $B r G R P$ genes. 


\section{Figures}

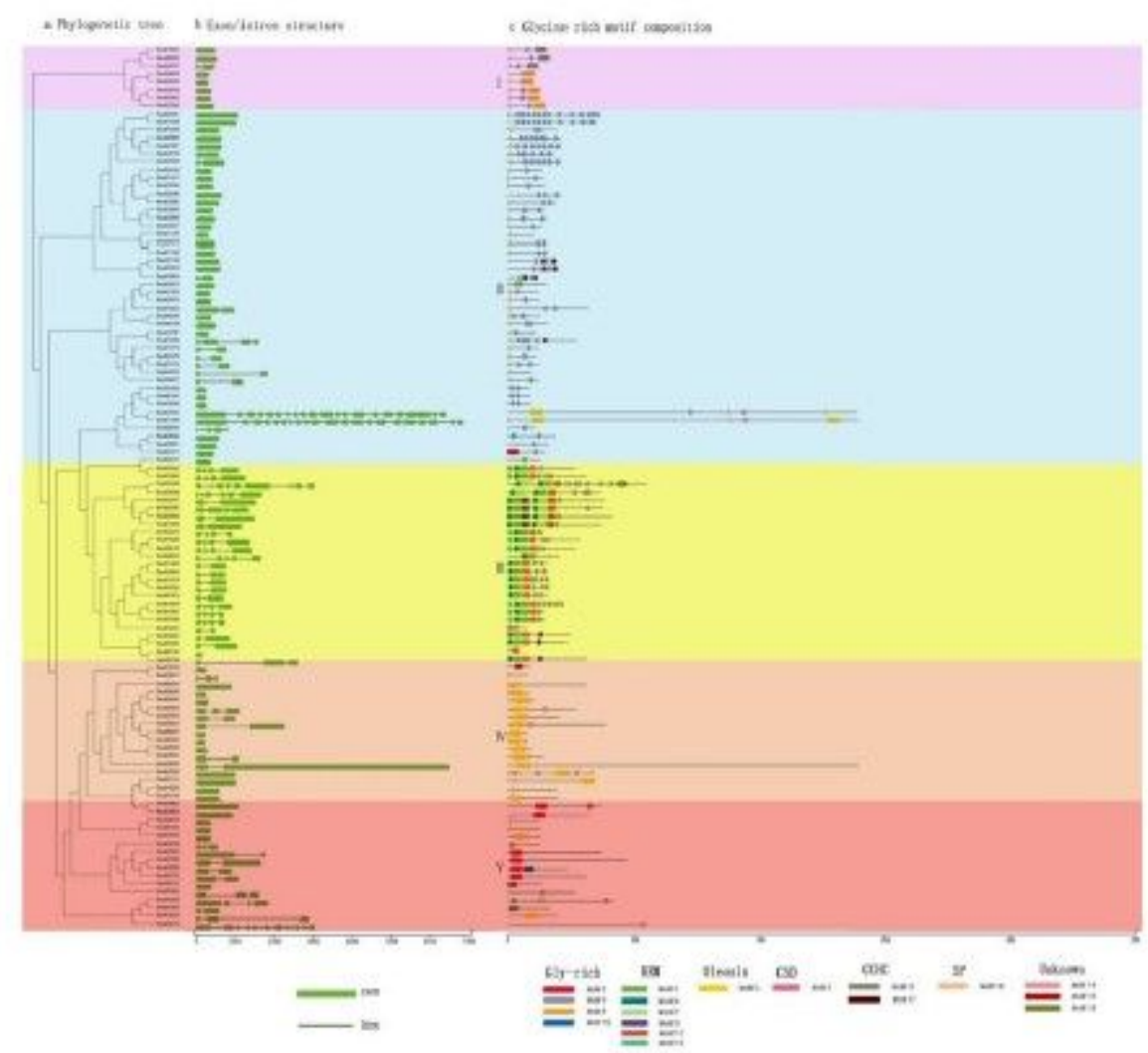

Figure 1

Phylogenetic relationship, gene structure and conserved structural composition of 110 BrGRP genes in Chinese cabbage. (a) Phylogenetic tree, by using Clustal $X$ to align the amino acid sequence, and using MEGA X to generate a phylogenetic tree by a contiguous method with a calibration parameter of 1000 . (b) the sequence structure distribution of BrGRP genes, the green box represents the exon, while the black line represents the intron, Scale indicates $1.0 \mathrm{~kb}$. (c) Schematic diagram of the conserved GRP protein motif in Chinese cabbage predicted by MEME, colored boxes indicate different motifs, black lines indicate nonconservative sequences, and scale bars represent 500 aa. 


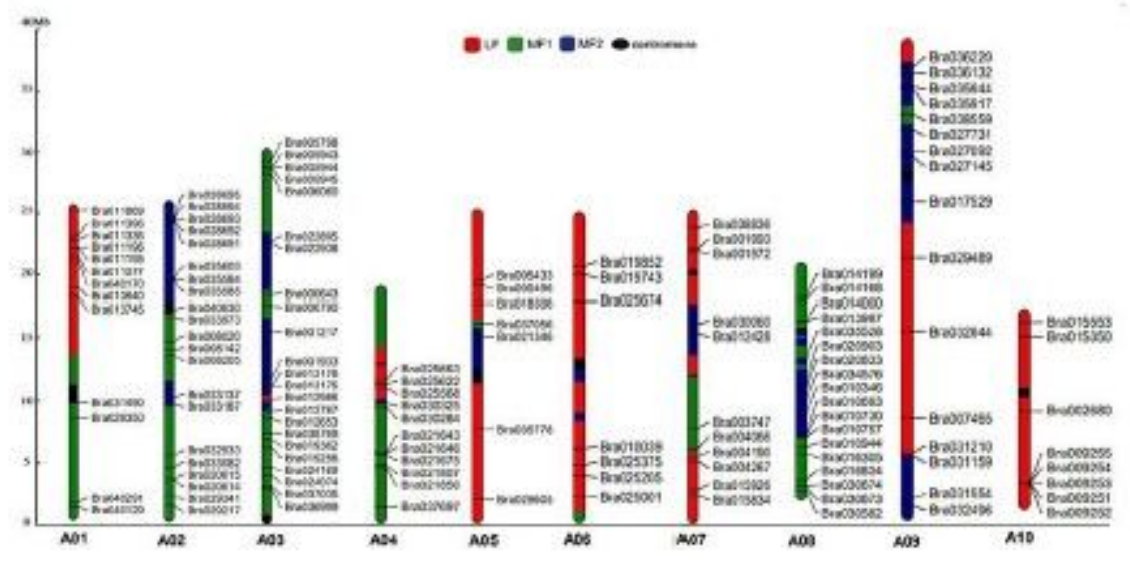

\section{Figure 2}

Chromosome distribution of BrGRP genes in Chinese cabbage. LF: the least fractionated subgenome; MF1: the medium fractionated subgenome; MF2: the most fractionated subgenome; centromere: position of chromosome centromere.

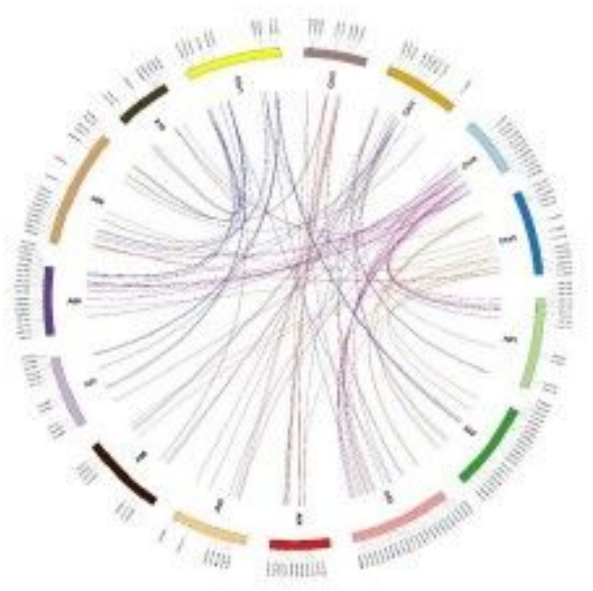

\section{Figure 3}

Syntenic analysis of GRP genes in Chinese cabbage, Arabidopsis thaliana. The orthologous and paralogous GRP genes are localized on the chromosomes of Chinese cabbage (A01-A10) and Arabidopsis thaliana (Chr1-Chr5) 
a

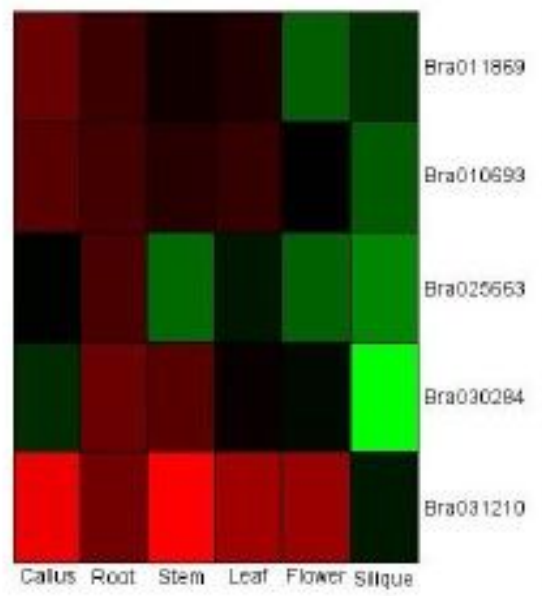

b

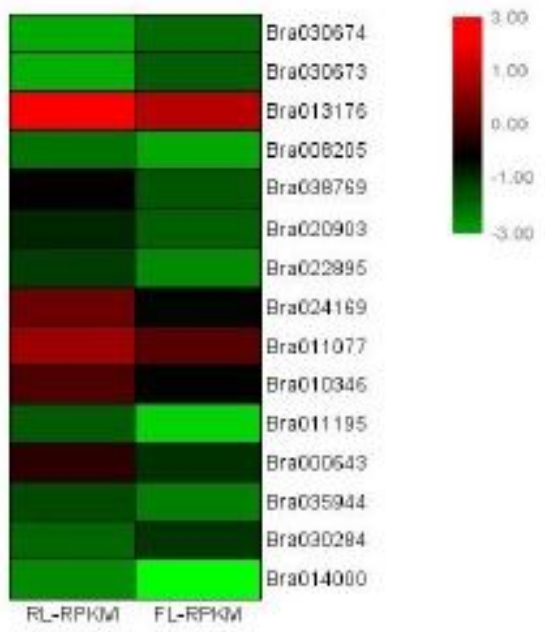

\section{Figure 4}

Expression analysis of BrGRP gene expression in Chinese cabbage. a: Differential expression pattern of BrGRP gene in different tissues of Chinese cabbage; $b$ : Differential expression pattern of BrGRP gene of Chinese cabbage in the rosette and folding leaves. 

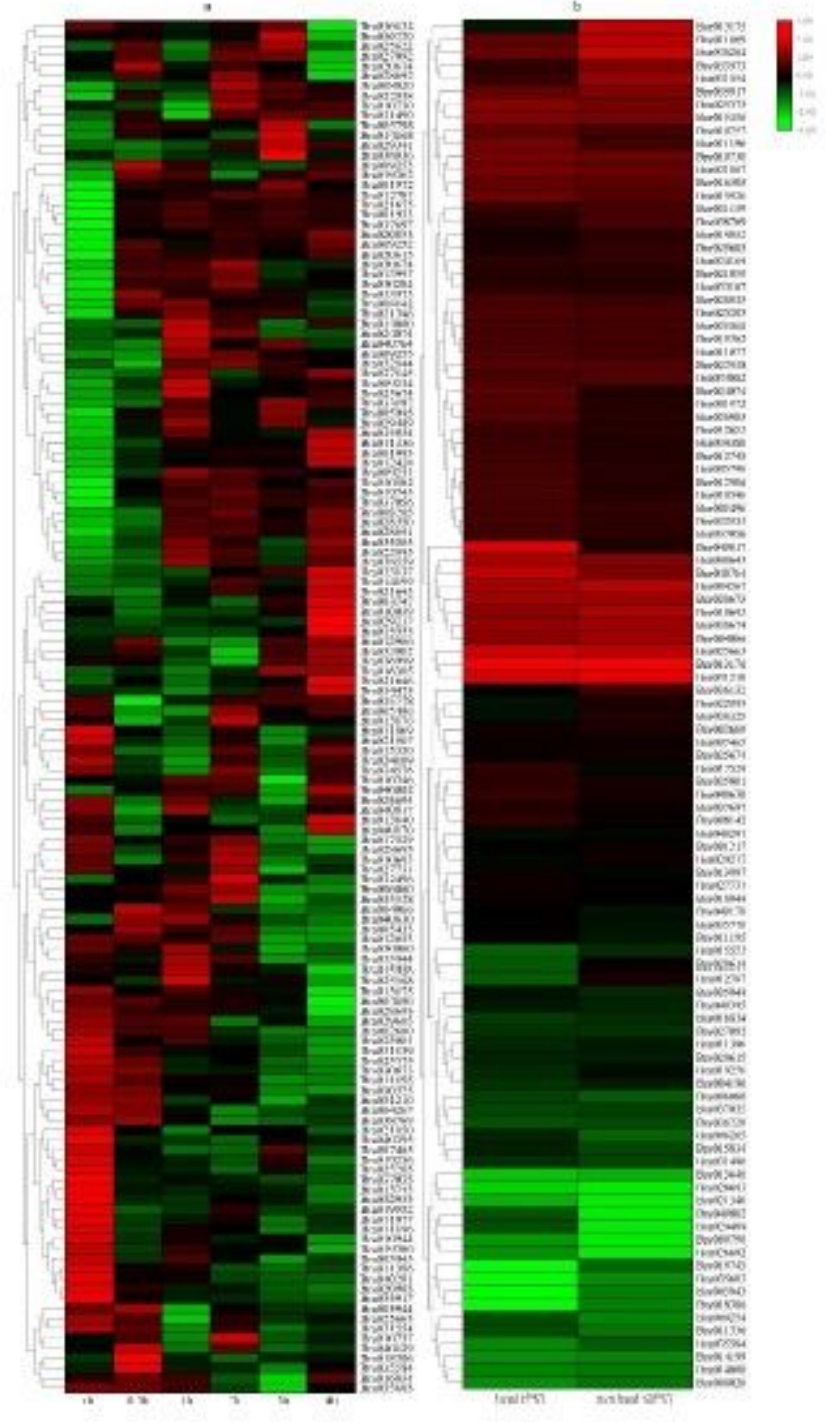

\section{Figure 5}

Expression analysis of BrGRP genes in Chinese cabbage. a: Differential expression pattern of BrGRP gene in Chinese cabbage under $45 \otimes$ heat stress for $0.5 h, 1 h, 2 h, 3 h$, and $4 h$; b: Differential expression pattern of BrGRP gene in Chinese cabbage under low-temperature stress at $25^{\circ} \mathrm{C}$ and $4{ }^{\circ} \mathrm{C}$. 

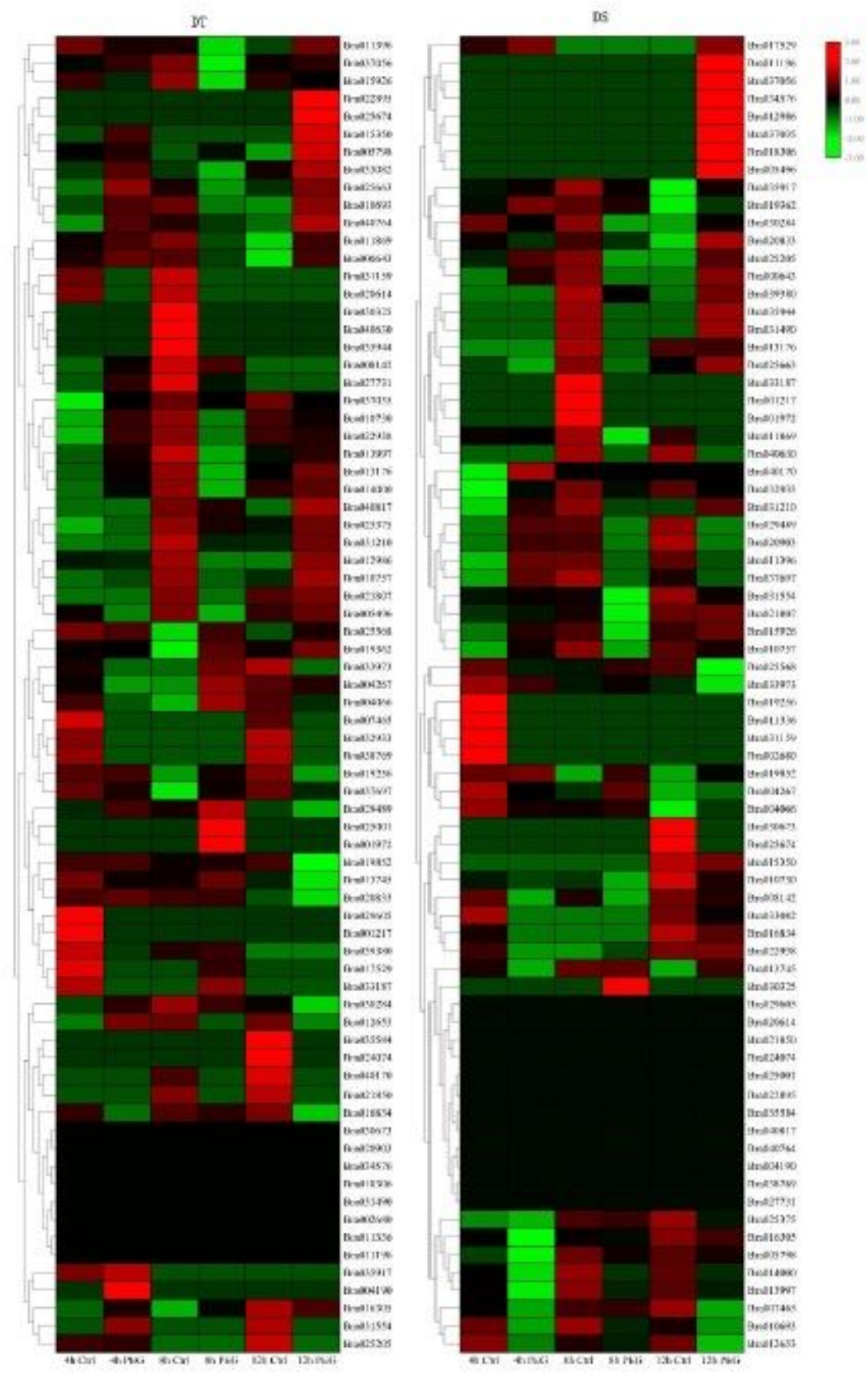

\section{Figure 6}

Differential expression profiles of BrGRP genes in drought-sensitive (DS) and drought-tolerant (DT) control and drought stress groups of Chinese cabbage at $4 \mathrm{~h}, 8 \mathrm{~h}$ and $12 \mathrm{~h}$. 


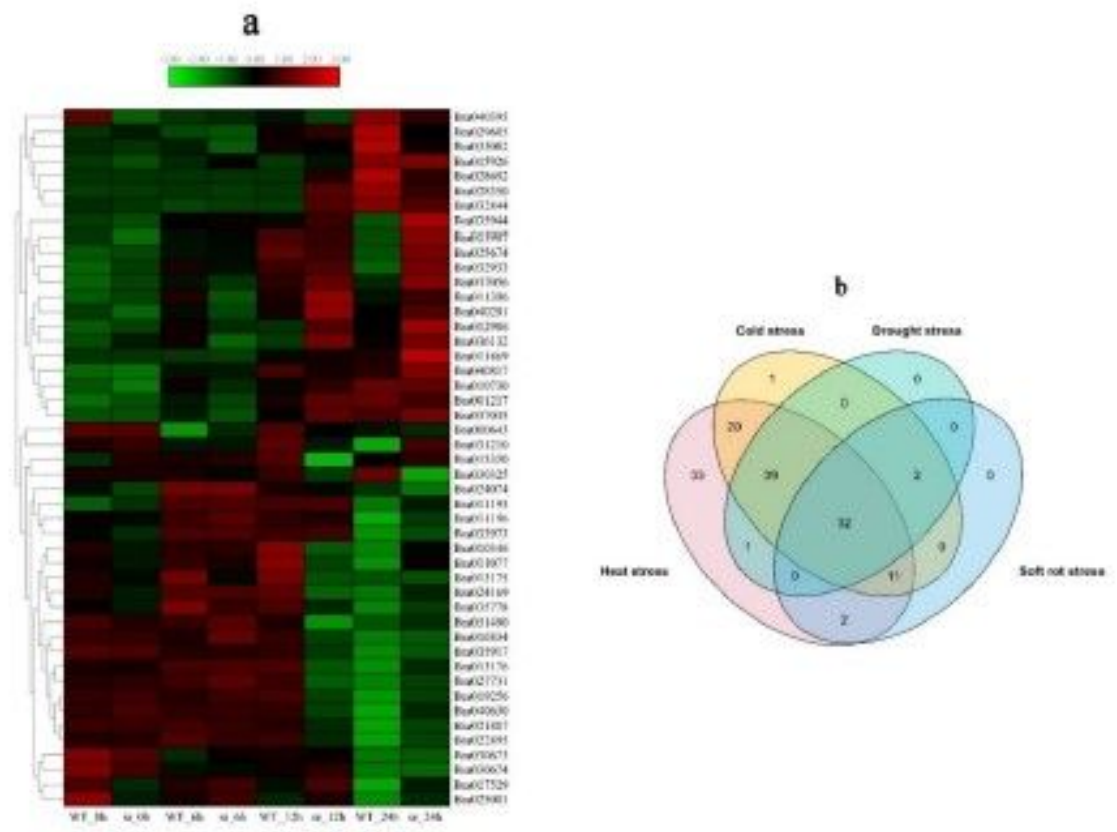

Figure 7

Expression analysis of BrGRP genes under soft rot stress (a) and the numbers of BrGRP genes involved in various stresses showed by Venn diagram (b). Fig.

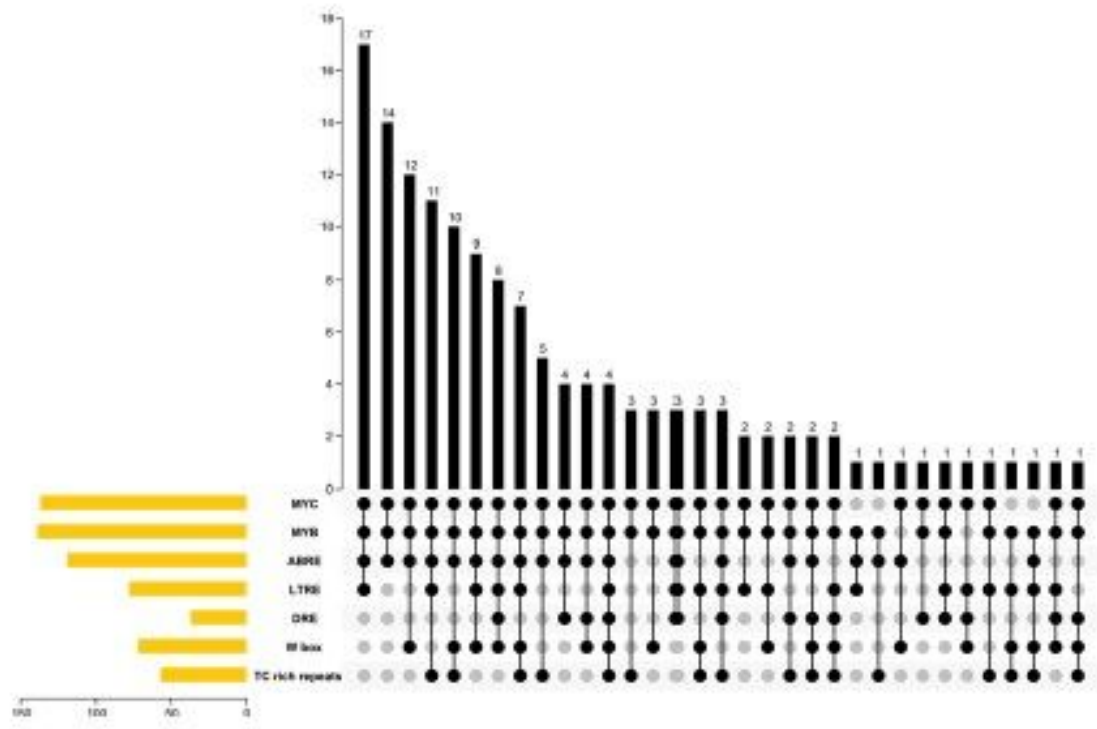

\section{Figure 8}

The numbers of the stress-related cis-acting elements in the promoter regions of BrGRP genes showed by Venn diagram. MYC (CANNTG), MYB (C/TAACNA/G), ABRE (ABA-responsive element, ACGT), LTR (lowtemperature-responsive element, CCGAAA), DRE (CCGAC), W box(TTGACC), TC rich repeats(GTTTTCTTAC). 


\section{Supplementary Files}

This is a list of supplementary files associated with this preprint. Click to download.

- Additionalfile5TableS14.xlsx

- Additionalfile2FigureS1.jpg

- Additionalfile1TableS1S3.xIsx

- Additionalfile4TableS13.xlsx

- Additionalfile3TableS4S12.xIsx 